\title{
Review
}

\section{Mind/Body Psychological Treatments for Irritable Bowel Syndrome}

\author{
Bruce D. Naliboff ${ }^{1,2,3}$, Michael P. Fresé ${ }^{1,2,3}$ and Lobsang Rapgay ${ }^{2}$
}

\author{
${ }^{1}$ UCLA Center for Neurovisceral Sciences and Women's Health, ${ }^{2}$ Department of Psychiatry and Biobehavioral \\ Sciences, David Geffen School of Medicine at UCLA and ${ }^{3}$ Veterans Administration Greater Los Angeles Healthcare \\ System, Los Angeles, CA, USA
}

\begin{abstract}
Currently, the goal of treatment for those with irritable bowel syndrome (IBS) is to improve the quality of life through a reduction in symptoms. While the majority of treatment approaches involve the use of traditional medicine, more and more patients seek out a non-drug approach to managing their symptoms. Current forms of non-drug psychologic or mind/body treatment for IBS include hypnotherapy, cognitive behavioral therapy and brief psychodynamic psychotherapy, all of which have been proven efficacious in clinical trials. We propose that incorporating the constructs of mindfulness and acceptance into a mind/body psychologic treatment of IBS may be of added benefit due to the focus on changing awareness and acceptance of one's own state which is a strong component of traditional and Eastern healing philosophies.
\end{abstract}

Keywords: irritable bowel syndrome - mind body interactions - mindfulness - psychological treatments - stress response

\section{Introduction}

Irritable bowel syndrome (IBS) is the most common of the functional gastrointestinal disorders $[10-20 \%$ prevalence (1)] and has an impact on quality of life comparable to that of depression and chronic renal failure (2). IBS is characterized by chronic abdominal pain or discomfort associated with altered bowel habits. The human and economic costs of IBS and related functional bowel disorders (FBD) are very high. Affected individuals have more hospitalizations, work absenteeism and physician visit rates than the general population $(3,4)$. These disorders have been ranked as the second most common cause of illness related work absenteeism aside from the common cold $(5,6)$. FBD are the most frequent gastrointestinal (GI) diagnoses in primary care [ $>12 \%$ of primary care practice $(7,8)]$, represent $41 \%$ of patients seen by gastroenterologists (9) and account for

For reprints and all correspondence: Bruce D. Naliboff, PhD, Center for Neurovisceral Sciences and Women's Health, VAGLAHS, Bldg. 115, Rm. 223, 11301 Wilshire Boulevard, Los Angeles, CA 90073, USA. Tel: (310) 268-3242; Fax: (310) 794-2864;

E-mail: naliboff@ucla.edu
3.5 million physician visits and 2 million prescriptions each year (10). Partially due to an increased rate of unnecessary tests and procedures, patients with FBD account for 8 billion dollars in annual health care costs in the U.S. alone $(5,6)$.

\section{IBS from a Mind/Body Perspective}

As with some, but not all, of the functional disorders, IBS has a higher prevalence among women (about $2: 1$ ) even after accounting for potentially confounding psychologic and health care seeking variables. Recent survey studies (11) have also shown that IBS co-occurs with a high frequency along with other functional GI disorders (such as dyspepsia), it has a high co-occurrence with affective disorders even in persons not seeking health care and that the majority of increased health care costs associated with IBS are for non-GI complaints. Taken together, these descriptive data have suggested that at least for the majority of patients identified with IBS a biopsychosocial or mind/body model may be the most appropriate way to help understand the development, 
maintenance and treatment of IBS symptoms $(12,13)$. This is not to say that peripheral changes such as altered motility, bacterial overgrowth or heightened visceral sensitivity are not relevant for symptom generation in individual IBS patients, but instead that a comprehensive model of the disorder must try and account for how these abnormalities come about and persist despite normal homeostatic correction mechanisms.

\section{Stress and the Emotional Motor System (EMS)}

It is becoming, increasingly accepted that the enteric nervous system (sometimes called the 'second' or 'little' brain due to its extensive intraconnected neural network) is exquisitely monitored and modulated by the central nervous system, although most of this processing is not conscious. We and others have proposed that enhanced responsiveness of central stress circuits to exteroceptive (psychosocial) as well as interoceptive (visceral) stressors is a plausible pathophysiologic model for IBS (14). As illustrated in Figure 1, enhanced output of the EMS can explain the cardinal symptoms of IBS: altered autonomic balance results in altered bowel habits, stress-induced hyperalgesia results in abdominal pain and discomfort and enhanced activation of ascending arousal systems result in hypervigilance towards sensory stimuli. In addition to the hyper-responsiveness of the EMS to actual stressors, conditioned fear responses and cognitive factors play an important role in the chronicity of IBS symptoms. For example, selective attention to an increased threat appraisal of visceral sensations and conditioned GI symptom-related fear may maintain symptoms in the absence of actual stressors. As readers of this journal are well aware, central and peripheral systems are in constant interplay and affective and cognitive actions have peripheral consequences including changes in motility and peripheral immunity, which in turn provide important feedback to the brain that helps shape ongoing mental activity $(11,15)$.

A variety of experimental and clinical studies support the model described above. In general, IBS patients show altered perception of visceral events that is characterized by hypervigilance, hypersensitivity and increased autonomic arousal $(16,17)$. While peripheral GI factors may play a role in subsets of patients with IBS (e.g. postinfectious IBS), there is converging clinical and neurobiologic research to suggest that enhanced central stress responsiveness involving anxiety may provide a specific mechanism for enhanced visceral sensitivity found in these disorders (11). GI symptom-specific anxiety may be an especially important variable leading to increased pain sensitivity, hypervigilance and poor coping $(18,19)$. Mild psychologic stress increases visceral perception in IBS patients but not in healthy controls (20) and IBS symptoms are exacerbated following stressful life events (21). We have also recently shown that visceral-specific anxiety appears to be particularly important in IBS and may be the primary element in mediating the impact between changes in symptom severity and changes in quality of life in IBS sufferers (18). Recent brain imaging findings have now begun to show the central circuitry that may underlie many of the observations described above (22). For example, patients with IBS during visceral stimulation show increased activation in the anterior cingulate cortex, a brain region involved in vigilance and discomfort during physical and social situations (23).

In a recent article in this journal, Tan et al. (24) discussed the overlap of a Western biobehavioral model for disorders like IBS that incorporates the EMS and traditional Chinese medicine approaches to disease. In both, internal and external stressors are managed by a variety of overlapping physiologic systems; but when these systems are overwhelmed, physiologic breakdowns occur. As will be discussed below, there are also very similar complementary and overlapping Eastern and Western approaches to psychologic healing, although the language and theory may at first appear quite different.

\section{Treatment Themes Derived From Mind/Body Models}

Of particular relevance to the understanding of mind/ body psychologic therapies applied to IBS is the development of specific mind/body themes that have emerged from the research literature described above. These include the following:

(i) The presence of an increased stress response, especially an increased stress response associated with viscerally related events. Life stress can stimulate colon spasms, resulting in abdominal discomfort at stressful times. Whereas people might experience 'butterflies' or cramps when they are nervous or upset, a person with IBS will experience a higher level of distress, as their colon can be overly responsive to the smallest amount of conflict or stress. Whether through progressive muscle relaxation, meditation, yoga, counseling or changes to the stressful situations we face daily, reducing the level of stress in one's life can have a positive effect. In addition to life stress for those with IBS, visceral sensations and the context in which these sensations occur can be an anxiety producing experience. For patients with urgency and diarrhea, the anticipation of not making it to the bathroom on time can be unbearable. How an individual's symptoms are perceived, evaluated, and acted upon can determine illness behavior. The slightest amount of abdominal pain can lead to catastrophizing the experience and can result in 


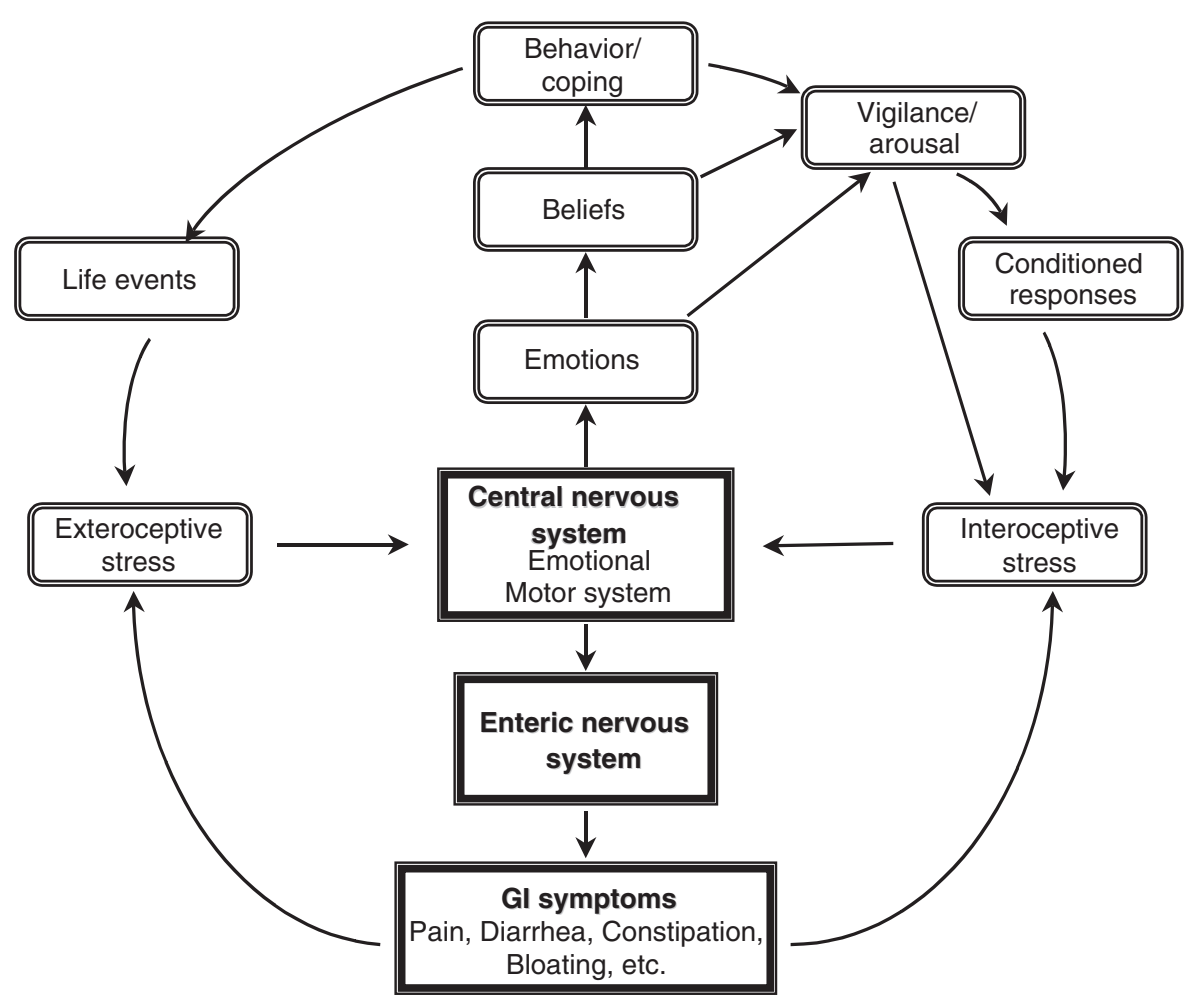

Figure 1. Mind/body interactions and IBS. The central nervous system and the enteric nervous system are in constant communication with a goal of efficient function and balance in the presence of internal and external stressors. The Emotional Motor System (EMS) refers to a parallel set of outputs from limbic and paralimbic circuits in the central nervous system, which generate distinct patterns of bodily responses associated with emotions (fear, anger, joy, etc.). These outputs occur in the form of autonomic nervous system responses, sensory modulation and HPA axis responses that have a powerful influence on the enteric nervous system. Feedback from the body to the EMS in the form of afferent nerve signals and neuroendocrine signals modify EMS responses. Ascending outputs to cortical regions of the brain generate patterns of vigilance, arousal and attention. Activation of the EMS may result from both internally generated stressors like those from GI symptoms (interoception) or externally generated stressors and may be the result of acutely occurring events or the result of conditioned responses, anticipation or beliefs. Especially important for understanding the relationship between stress and GI sensations is the fact that conscious perception may or may not be associated with activities of the EMS (11).

increased anxiety and possibly more symptoms. Anxiety can serve as part of a vicious cycle that involves a trigger (physical symptom such as abdominal pain), a reaction (anxiety) and a belief ('I am going to have diarrhea and miss my appointment'). Certain symptoms can cause more anxiety than others; for example, dining at a restaurant and experiencing severe gas pain might cause a person so much anxiety that they get up and leave. Similar mechanisms may also occur with sensations associated with constipation such as bloating.

(ii) Poor or inappropriate coping responses to GI-related events. Patients with IBS may develop hypervigilance to visceral sensations as well as avoidance of many pleasurable activities for fear of triggering symptoms. Poor coping responses, in part due to catastrophic beliefs and a poor sense of control over symptoms, play a critical role in mediating the relationship between affective states such as depression, severity of symptoms (e.g. pain) and real life limitations caused by IBS (25). Directly changing coping responses and beliefs in order to influence both mood and symptoms is a hallmark of certain behavioral interventions.

(iii) Psychosocial fears and isolation; including low selfesteem, depression and social withdrawal. IBS can take its toll on friendships, marriages, employment and one's sex life. People with IBS can become pre-occupied with fears of soiling themselves in public and as a result, isolate themselves and only venture out when necessary. If employed, many are afraid to eat while at work out of fear that they might become incapacitated with pain and/or multiple bowel movements. Shame and guilt, as well as anger, are common emotions described by those with IBS, as they are relegated to keeping their disorder a secret out of embarrassment. As the isolation is compounded, many patients with IBS develop depression and anxiety, which serves to further complicate the presentation of IBS. 
Although derived from the clinical literature, these three themes can also be viewed in the context of the evolving physiologic model presented above. Hypersensitivity to stress manifested by increased limbic system reactivity can be directly associated with increased perception of and responses to visceral sensations via inappropriate up-regulation of pain facilitation systems. Cognitive activity plays an important role in modulation of limbic activation via cortico-limbic interactions and behaviors such as avoidance may perpetuate arousal responses by negating normal inhibition in these circuits (26).

\section{Psychologic Treatments Applied To IBS}

Three types of psychologic treatment modalities have received the most attention in the literature to date. These include several forms of cognitive behavioral therapy (CBT), a brief form of insight-oriented psychotherapy, and a gut-directed form of hypnotherapy. We will review each of these and then discuss several newer, less well-studied forms of mind/body intervention that may also hold promise for IBS. All of these treatments address the major themes discussed above but they differ substantially in their emphasis. Following a brief discussion of the rationale for each type of therapy, we will examine the growing empirical literature on the efficacy of psychologic interventions for IBS and their possible mechanisms of action.

\section{CBT}

The first generation of researchers took a wide range of existing psychotherapies and behavioral strategies that were originally designed to treat other disorders and adapted and combined them to develop protocols to treat IBS. The therapies that were used in these protocols included a variety of relaxation exercise procedures including progressive muscle relaxation, breathing exercises, autogenic training, cued relaxation and simple forms of meditation. These were combined with cognitive exercises to change irrational and/or maladaptive beliefs regarding one's illness (e.g., 'it will never change'), or poor self-efficacy, (e.g., 'I cannot function another day'), as well as interpersonal stressors (e.g., 'everyone thinks I am strange'). Treatments also included a strong presentation of a biopsychosocial model to give patients a new way to understand their symptoms in relationship to stress and to look for ways to better manage symptoms rather than cure the 'disease'. Patients are also typically taught to gradually change from avoidant, unassertive, illness driven behaviors to healthier, active and assertive behaviors including increasing exercise, exploration of previously avoided interpersonal situations and shedding a sick role identity.

CBT is typically applied in a 10-12 individual session format and while the above elements are included in most protocols the emphasis may vary across providers and institutions as well as theoretical frameworks. For example, some approaches may emphasize managing life stress while others may place more emphasis on changing responses to interoceptive sensations or symptom-specific anxieties. Group formats are also used and may have some advantages in terms of efficiency and support (27).

CBT treatments for IBS have been the most studied and a number of controlled trials have been published. Overall, CBT has been found to be significantly more effective than usual IBS care. In the most recent and best-controlled study to date, a large group of women with IBS were randomly assigned to either 12 weeks of CBT or an educational control condition. CBT led to a positive response rate that was much higher ( 70 versus $37 \%$ ) than the control group on a composite outcome including treatment satisfaction, symptoms and quality of life (28). Of particular note, CBT was also superior to another group receiving a tricyclic antidepressant medication.

\section{Brief Psychodynamic Psychotherapy}

There is a long history of psychodynamically based therapies being applied to somatic disorders including functional GI disorders. In fact, many of the classic psychoanalytic cases were treated for functional symptoms. The brief version of psychodynamic psychotherapy that has been empirically studied in IBS is an insightoriented talk therapy that focuses on in-depth discussion of symptoms, emotions and exploration of the links between symptoms and emotional conflicts. Help with identifying and resolving interpersonal conflicts that may contribute to stress and symptoms is also an important focus of treatment.

This form of psychotherapy has been used primarily in the UK. A series of positive trials have shown that brief psychodynamic psychotherapy is effective when compared to usual care and supportive listening. For example, Svedlund et al. (5) randomized 101 outpatients with IBS into two treatment groups; both of which received the same medical treatment, with one group also receiving 10 one-hour sessions of dynamically oriented individual psychotherapy, spread over 3 months. After 3 months, there was a significant improvement in somatic symptoms in the group that received the individual psychotherapy. Further, at one year follow up, the patients who received the individual psychotherapy continued to show further improvement, while the patients who only received the medical treatment showed some deterioration. These findings suggest that the combination of medical treatment and dynamic psychotherapy improves outcome both in the short term and long run.

In the most recent and largest study, Creed et al. randomized 257 patients to either eight-session psychodynamic interpersonal therapy, a selective serotonin 
reuptake inhibitor antidepressant (paroxetine) or standard medical care (29). There was no significant therapeutic effect on symptoms for either psychotherapy or medication compared with standard care at the end of treatment or at follow-up. However, both treatment groups improved significantly on quality of life and the psychotherapy group had lessened health care costs compared with the other groups at follow-up. Thus, it appears that this brief, traditional talk therapy can also be helpful for IBS at least in terms of global outcomes.

\section{Hypnotherapy}

There has been a long tradition of using hypnotherapy for the treatment of medical conditions. There are a variety of forms of hypnotherapy used in psychologic and medical treatment, but in IBS the primary form has followed a 7-12 session gut-directed hypnotherapy protocol initially developed by Whorwell $(30,31)$. Gut-directed hypnotherapy involves two people initially, one of whom is inducing the hypnotic state and then offering specific suggestions for improved GI functioning. The clinical steps of the protocol involved: (i) the induction of overall physical relaxation with progress in muscle relaxation, (ii) ego strengthening using metaphors such as, the willow tree and (iii) gut-specific relaxation suggestion induced by placing one's warm hands on the gut. Ego strengthening is a psychologic exercise which fosters inner strength through sentence completion and visualization tasks (32). Suggestions are made to reduce fear of pain and discomfort and of pre-occupation with sensation in the gut. The final phase involves imagery to increase one's sense of control and self-efficacy over IBS symptoms.

In a recent review of the hypnotherapy literature in IBS, Whitehead reviewed 11 studies including five controlled trials (33). There were significant difficulties with most of the studies in terms of sample size and type of control conditions, but this review did conclude that the literature supports hypnosis to have a substantial positive impact on IBS, even for patients unresponsive to standard medical interventions. The median response rate was $87 \%$ and bowel symptoms generally improved by about $50 \%$, as did psychologic and quality of life variables. The gains appeared to be long lasting as with the other psychologic treatments discussed earlier. It should also be noted that the hypnosis literature has not identified hypnotizability as a critical factor in outcome. As with other psychologic treatments the mechanism by which hypnotherapy works has not been established. Changes in visceral sensitivity were initially reported for this treatment but not consistently across studies.

\section{Reviews of Clinical Trial Outcomes}

It is clear from the above discussion that studies of each of the individual psychologic interventions appear to yield positive treatment effects. This is also the general conclusion of several systematic reviews that have examined the overall psychologic treatment literature in IBS. For example, a recent meta-analysis of controlled trials of psychologic treatments for IBS $(6,34)$ concluded that psychologic treatments for IBS as a class are effective and seem to be as effective for changing bowel symptoms as for changing mood. The American College of Gastroenterology has also performed a review of the empirical literature of treatments for IBS and concluded that 'Behavioral therapy is more effective than placebo at relieving individual IBS symptoms' (35). Several other reviews have also concluded that psychologic interventions are generally effective although not universally so $(36,37)$.

\section{Mechanisms of Treatment Effects}

While psychologic mind/body interventions have proven to be among the most effective treatments for IBS it is not at all clear by what mechanism they work, which treatments might be best for specific patient subgroups or if combinations of treatments may yield better results than single interventions alone. It is known that these interventions can be effective for patients both with and without psychiatric co-morbidity and that positive outcomes are not completely accounted for by changes in mood (38). It has been suggested that psychologic treatments aimed at functional disorders may have their primary impact on illness-related cognitions and behaviors and not on distress. These may include improved ability to cope, increased perception of control, reduced avoidant and increased healthy behaviors and reduced pre-occupation and amplification of bodily symptoms. Although treatment mechanisms have been tested primarily through self-report measures to date, a recent study has examined functional brain imaging data from a small group of patients before and after CBT during baseline and visceral stimulation (39). The results suggest in a preliminary way that CBT may improve IBS symptoms through cortical modulation of limbic and pontine circuitry associated with vigilance and pain modulation. These responses are consistent with some of the same circuitry involved in placebo modulation of pain as well as acute changes in perception during hypnosis and anxiety.

\section{New Approaches}

Although the current mind/body IBS treatments, especially CBT and hypnotherapy have been shown to be effective compared to standard medical care, the symptom changes in many cases are only moderate or inconsistent across subjects. Other modalities, especially those that may work better for patients not interested in or amenable to these therapies would be desirable. 
Two alternative forms of treatment that may be useful due to their rather unique focus on changing awareness and acceptance of one's own state are mindfulness and acceptance.

\section{Mindfulness Meditation}

As discussed above, relaxation exercises are a common component of many of the CBTs for IBS. The relaxation response is a derivative of Eastern practices of meditation and adapted for the convenience of a Western population. The focus of the practice involves four main components: selecting a quiet environment, assuming a comfortable position, focusing on the word 'one' or any other meaningful word and maintaining a passive attitude. However, relaxation response differs in some respects from traditional meditation and in particular from mindfulness practice, which has been shown to be efficacious for a wide range of medical and psychiatric conditions as well. While mindfulness demands an active, yet relaxed state of consciousness, relaxation response suggests a passive attitude. Passive relaxation focuses on the entire body and a general state of calmness. According to mindfulness practice, all meditation practice requires an active state of consciousness, since passivity would facilitate an overly relaxed state of consciousness that would make it difficult to develop attention and awareness, the core basis of mindfulness. While these processes are involved in some form in relaxation response, they are not the basis and focus of the meditation practice as they are in mindfulness.

Over the last few decades, mindfulness (primarily in the form of Mindfulness Based Stress Reduction, MBSR) has received increasing attention from clinicians and researchers as a stand-alone intervention or as an integrated component of CBT. The promising outcomes of the initial pilot studies for chronic medical and psychiatric conditions are now being validated by randomized controlled studies which demonstrate an impressive reduction in psychologic morbidity associated with chronic medical conditions (40-42).

According to a consensus among Bishop and a group of researchers, the key defining feature of mindfulness is sustained attention/awareness and acceptance (43). While CBT is primarily directed at changing contents such as distorted and maladaptive thoughts and feelings and replacing them with more realistic and adaptive experiences, mindfulness helps to replace habitual ways of relating to thoughts and feelings in a more non-reactive and accepting attitude (44). Teasdale et al. (44-46) speculate that mindfulness facilitates attention to cues, responses and contingencies in the present which enables change of rigid ways of responding to flexible ones. Wells suggests that mindfulness also helps to develop a meta-cognition that enables one to see that thoughts are not realities and thus reduce the preoccupations with the contents of the thoughts (47).

In an early non-controlled study on mindfulness for chronic pain (which included subjects with gastrointestinal pain), Kabat-Zinn demonstrated significant results during post treatment as well as follow-up (48). In a controlled study, Blanchard and Keefer (49) showed that relaxation response meditation was superior to the control group and significant improvement in flatulence and belching was observed at post-treatment within subjects.

Mindfulness facilitates the practice of engaged attention on a selected object such as the breath or a bodily sensation in a non-judgmental manner while being open to any thoughts, feelings, images or other sensations. When a thought or feeling presents itself, the engaged attention on the breath is let go and one shifts to a state of disengaged attention and observation of the new experience. The disengaged attention is then maintained for as long as necessary and then the experience is appropriately labeled. When the labeling is complete and there are no additional distractions, the subject pauses in stillness before reverting to engaged attention. The purpose is to train in focused attention and gain control and insight into the dynamics and processes of the mind. As the subject gains more attention skills and insight into the nature of the mind, the busy mind begins to quiet down and attention and observation skills become more refined. The subject is now able to increase awareness of the subtle processes of bodily sensations first and then, more importantly, mental activity, resulting in further deepening of insight and control over the workings of the mind.

During each sitting, the subject is trained to develop skills in the various mechanisms of mindfulness (i.e. the factors that account for the function of mindfulness). There are a number of primary mechanisms of mindfulness, including: (i) being in the present moment in a non-judgmental and accepting manner; (ii) letting go of a state of consciousness and shifting comfortably to experience the other; (iii) pausing in stillness and (iv) labeling mental activities systematically and accurately and exposure to immediate experiences. In applying mindfulness for the treatment of IBS, the patient is first trained in increasing engaged attention on an object while remaining open to automatic thoughts, sensations and feelings. When the patient has acquired basic skills of attention and observation, the patient is then trained to apply the various therapeutic functions of mindfulness in a structured and sequential manner to treat IBS symptoms. In the first phase, the patient is taught to be in the present moment of experience of sensations of IBS symptoms while being open in a non-judgmental manner to associated sensations, thoughts, and feelings. The purpose is to develop acceptance of the experience of IBS symptoms without judgment and in the present moment. 
As the patient is able to do so, he or she is trained to reduce past memories and, more importantly, future concerns and anxieties about IBS symptoms.

Mindfulness involves training in how to let go of an experience and shift comfortably to another. As the patient learns to observe the new experience with disengaged attention and observation, the patient then labels the experience. He or she then pauses in stillness in order to be open to any other mental activity that is occurring. Correct labeling of an experience is critical to the practice of mindfulness in that it ensures accurate insight into the workings of the mind. The subsequent pause in stillness provides an opportunity for the IBS patient to assess and decide his or her next process. And finally, the patient is then exposed to the painful sensation of IBS in order to increase his or her tolerance level of pain and suffering. As the patient learns to tolerate the exposure in a state of quiet observation, the patient's increased attention and observation facilitates greater awareness of and insight into the workings of the triggers and exacerbating and maintaining factors of IBS symptoms.

It is important to remember that mindfulness does not directly address many of the psychosocial factors associated with IBS. Consequently, mindfulness may be combined with CBT which is used to address maladaptive thoughts and beliefs, as well as pain behavior and functioning. In addition to using CBT to address these specific clinical issues, CBT is also used to address resistance to mindfulness practice such as issues with acceptance of pain. Once the patient is able to be in the present moment of experience in a non-judgmental and accepting manner, subsequent mindfulness processes are developed.

Though there are no direct studies of the efficacy of mindfulness for IBS, the fact that mindfulness has been shown in preliminary studies to be effective for chronic pain as well as other chronic medical conditions, suggests that it may be potentially beneficial for IBS. Given the EMS model presented, we would expect that mindfulness may be particularly beneficial in regulating enhanced responsiveness of central stress circuits to exteroceptive (psychosocial) as well as interoceptive (visceral) stressors. In addition, mindfulness practice by providing sustained but accepting attention on pain related experiences such as sensations of pain, fear of pain and avoidance of pain, may reduce the habitual pre-occupation with maladaptive mental content.

Another way in which mindfulness may help to reduce pain, distress and discomfort is by serving as an exposure intervention $(44,46)$. Unlike standard CBT exposure which is strictly based on a stimuli and response paradigm that requires conscious effort to inhibit certain responses and activation of opposing responses, mindful exposure involves a more flexible response process to stimuli by being experientially open without any efforts to inhibit or intentionally activate experiences. As a result, it is very likely that mindful exposure may reduce the resistance involving the use of covert coping strategies seen in standard CBT exposure. Mindfulness practice may also help to uncouple the sensory experience of pain and other somatic symptoms from the affective/evaluative alarm reaction and reduce the experience of suffering via cognitive reappraisals.

Since mindfulness has been shown to be effective in significantly reducing the risk for recurrence of depression and preliminary studies have show its promise for a wide range of anxiety disorders, it is therefore likely that mindfulness may also help with comorbid anxiety and depression that play a significant role in exacerbating and maintaining IBS. In a multi-center controlled study, Teasdale et al. (50) demonstrated that mindfulness plays a significant role in preventing the risk of relapse in recovered depressed patients and that attention to present moment training in attention is responsible for helping depressed patients decrease their over-general recall of past experiences. Furthermore, repeated dysphoric experiences in recovered depressed patients can activate overgeneral recall of past negativistic experiences that leads to risk of relapse (50).

Mindfulness training has also been used to change habitual worrisome patterns in anxiety patients by training them with an alternative perspective of experience that facilitates detachment from habitual ways of responding. Furthermore, the key feature of worry (e.g. the focus on future oriented concerns), can be potentially modified or reduced by focusing on present moment experiences (46). It is, therefore, likely that mindfulness may enhance the efficacy of CBT in treating IBS and comorbid conditions of anxiety and depression.

The mechanisms of mindfulness may therefore be highly relevant to treating many of the issues of IBS and could facilitate the efficacy of CBT. While CBT would be used to bring about change in the contents of the maladaptive thoughts, feared feelings and pain behavior, mindfulness would be effective and useful in changing the processing and self-regulation of thoughts, sensations, feelings and behavior related to IBS.

\section{Acceptance and Commitment Theory}

Another related therapeutic approach that may have utility for IBS is that of acceptance. Many individuals with IBS engage in a variety of experiential avoidance strategies, such as distraction or suppression of thoughts, feelings and bodily sensations, which serve to perpetuate IBS distress. For those suffering from anxiety, escape and avoidance serve to maintain and even elevate the anxiety. Some forms of CBT use graded exposure to help habituate anxiety and decrease avoidance. Acceptance and Commitment Therapy (ACT) takes its overall goal as that of increasing psychologic flexibility or the ability to 
'contact the present moment more fully as a conscious human being and to change or persist in behavior when doing so serves valued ends' (51). This flexibility is established through experiential exercises designed to activate core processes such as acceptance, being present, and committed action. ACT has shown promise as a treatment modality for disorders such as chronic pain, depression, eating disorders and stress. Mindfulness and acceptance offer both the therapist and the patient an opportunity to redefine both the problem and the solution and look promising as mind/body psychologic treatments for IBS.

ACT seeks to facilitate psychologic flexibility by focusing on present moment experiences and one's responses in order to change behavior based on personal values (52). Among the six processes that facilitate change, acceptance and cognitive defusion play a central role. Parallels between ACT and mindfulness have been shown (53) as ACT incorporates mindfulness from the perspective of the Relational Frame Theory (52). Preliminary studies have demonstrated that acceptance of chronic pain reduces pain, psychologic distress, physical and psychologic disability $(54,55)$; furthermore, McCracken and Eccleston (56) found that acceptance of chronic pain was a more effective predictor of pain, depression, anxiety and disability than measures of coping.

According to the fundamentals of ACT, worries and fears are produced along with the effort to avoid all aversive thoughts, feelings and somatic discomfort. Similar to mindfulness, acceptance (and cognitive diffusion treatments) encourages openness to (rather than inhibition of) experience, the contents and consequences of thoughts and feelings, while monitoring (e.g. neutral observation and labeling) the mental processes of thinking, feeling and sensing.

Unlike cognitive therapy, which seeks to process the irrational nature of thoughts, ACT uses exposure to both interoceptive as well as exteroceptive sensations of chronic pain (57). According to ACT, the target of exposure is the habitual manner in which the patient responds to pain rather than the contents of thoughts that are associated with pain (57). Such an approach based on acceptance has been shown to be effective for pain and a variety of other problems. $(58,59)$.

ACT has conceptual as well as therapeutic advantages over current psychologic models by virtue of one of the aims of ACT: to address the patient's concerns in a mindful compassionate way, while encouraging the patient to pursue what is important to him/her. Regarding the treatment of IBS, ACT will allow patients to learn to accept and live with their unwanted thoughts/worries/ bodily sensations and feel empowered by taking charge and moving their lives in directions which they value. ACT incorporates mindfulness techniques as well as acceptance strategies, along with specific behavioral approaches from CBT that are known to be effective.

While cognitive behavioral approaches are clearly beneficial for both understanding and treating IBS, ACT and mindfulness represent possible alternatives to the traditional CBT approach. We would posit that for patients with IBS, targeting the function of cognitions and emotions is an advantage over a more traditional CBT approach where the emphasis is on changing the form or frequency of psychologic events. Cognitions and emotions can have a deleterious effect on a patient's experience of their symptoms; for example, if a patient catastrophizes his symptoms, he believes he will not make it to the bathroom on time and his level of anxiety is elevated, his experience (in the moment) will be very different than if he has had some sort of therapeutic intervention, which targets the function of cognitions and emotions and in particular, the brain-gut connection.

\section{Conclusions}

It is clear that psychologic treatments which emphasize stress management, self-empowerment and positive coping have much to offer for patients with chronic medical conditions like IBS. From the traditional Western psychologic perspective, increased arousal, poor coping strategies and irrational beliefs may drive much of the suffering associated with these syndromes. Another perspective of the same imbalance is offered by traditional and Eastern systems. A very clear sign of the growing recognition of the overlap of these ideas and the importance of finding ways of integration is the increasing popularity among both patients and professionals of mindfulness- and acceptancebased therapies. These new approaches may hold particular promise for chronic medical syndromes since they focus on changing awareness and increasing acceptance of one's own state and increasing global balance beyond just specific symptom relief.

\section{Acknowledgments}

The authors would like to thank Teresa Olivas and Cathy Liu for their editorial assistance in the preparation of this manuscript. This research has been supported in part by P50 DK64539, R24 AT002681, NR 04881 and VA Medical Research.

\section{References}

1. Bradette M, Pare P, Douville P, Morin A. Visceral perception in health and functional dyspepsia. Crossover study of gastric distension with placebo and domperidone. Dig Dis Sci 1991;36:52-8.

2. Gralnek IM, Hays RD, Kilbourne A, Naliboff B, Mayer EA. The impact of irritable bowel syndrome on health-related quality of life. Gastroenterology 2000;119:654-60.

3. Drossman DA, Andruzzi E, Temple R. Work absenteeism, disability and health care use in a national sample of persons with functional GI disorders. Gastroenterology 1993;104:A499. 
4. Drossman DA, Li Z, Andruzzi E, Temple R, Talley NJ, Thompson WG, et al. U.S. householder survey of functional gastrointestinal disorders. Prevalence, sociodemography and health impact. Dig Dis Sci 1993;38:1569-80.

5. Svedlund J, Sjodin I, Ottosson J-O, Dotevall G. Controlled study of psychotherapy in irritable bowel syndrome. Lancet 1983;2:589-92.

6. Blanchard EB, Schwarz SP, Suls JM, Gerardi MA, Scharff L, Greene B, et al. Two controlled evaluations of multicomponent psychological treatment of irritable bowel syndrome. Behav Res Ther 1992;30:175-89.

7. Drossman DA. Clinical research in the functional digestive disorders. Gastroenterology 1987;92:1267-9.

8. Mendeloff AL. Epidemiology of functional GI disorders. In: Chey, WY, ed. Functional Disorders of the Digestive Tract, Raven Press: NewYork 1983; 9-13.

9. Mitchell CM, Drossman DA. Survey of the AGA membership relating to patients with functional gastrointestinal disorders. Gastroenterology 1987;92:1282-4.

10. Sandler RS. Epidemiology of irritable bowel syndrome in the United States. Gastroenterology 1990;99:409-15.

11. Mayer EA, Craske MG, Naliboff BD. Depression, anxiety and the gastrointestinal system. J Clin Psychiatry 2001;62:28-36.

12. Drossman DA. Presidential address: Gastrointestinal illness and the biopsychosocial model. Psychosom Med 1998;60: 258-67.

13. Naliboff BD, Munakata J, Chang L, Mayer EA. Toward a biobehavioral model of visceral hypersensitivity in irritable bowel syndrome. J Psychosom Res 1998;45:485-92.

14. Mayer EA. Emerging disease model for functional gastrointestinal disorders. Am J Med 1999;107:S12-S19.

15. Damasio AR. Descartes' Error. New York: G.P. Putnam's Sons, 1994.

16. Naliboff BD, Munakata J, Fullerton S, Gracely RH, Kodner A, Harraf F, et al. Evidence for two distinct perceptual alterations in irritable bowel syndrome. Gut 1997:41:505-12.

17. Tillisch K, Mayer EA, Labus JS, Stains J, Chang L, Naliboff BD. Sex-specific alterations in autonomic function among patients with irritable bowel syndrome. Gut 2005;54:1396-401.

18. Labus J, Bolus R, Chang L, Wiklund I, Naesdal J, Mayer E, et al. The visceral sensitivity index: Development and validation of a gastrointestinal symptom-specific anxiety scale. Aliment Pharmacol Ther 2004;20:89-97.

19. Hazlett-Stevens H, Craske MG, Mayer EA, Chang L, Naliboff BD. Prevalence of irritable bowel syndrome among university students: the roles of worry, neuroticism, anxiety sensitivity and visceral anxiety. J Psychosom Res 2003;55:501-5.

20. Dickhaus B, Mayer EA, Firooz N, Stains J, Conde F, Olivas TI, et al. Irritable bowel syndrome patients show enhanced modulation of visceral perception by auditory stress. Am $J$ Gastroenterol 2003;98:135-43.

21. Bennett EJ, Tennant CC, Piesse C, Badcock CA, Kellow JE. Level of chronic life stress predicts clinical outcome in irritable bowel syndrome. Gut 1998;43:256-61.

22. Chang L. Brain responses to visceral and somatic stimuli in irritable bowel syndrome: A central nervous system disorder? Gastroenterol Clin North Am 2005;34:271-9.

23. Naliboff BD, Derbyshire SWG, Munakata J, Berman S, Mandelkern M, Chang L, et al. Cerebral activation in irritable bowel syndrome patients and control subjects during rectosigmoid stimulation. Psychosom Med 2001;63:365-75.

24. Tan S, Tillisch K, Mayer EA. Functional somatic syndromes: emerging biomedical models and Traditional Chinese Medicine. Evid Based Complement Alternat Med 2004;1:35-40.

25. Lackner JM, Quigley BM, Blanchard EB. Depression and abdominal pain in IBS patients: the mediating role of catastrophizing. Psychosom Med 2004;66:435-41.

26. Mayer EA, Naliboff BD, Craig AD. Neuroimaging of the brain-gut axis: from basic understanding to treatment of functional GI disorders. Gastroenterology 2006;131:1925-42.

27. Toner BB, Segal ZV, Emmott S, Myran D, Ali A, DiGasbarro I, et al. Cognitive-behavioral group therapy for patients with irritable bowel syndrome. International Journal of Group Psychotherapy 1998;48:215-43.
28. Drossman DA, Toner BB, Whitehead WE, Diamant NE, Dalton CB, Duncan S, et al. Cognitive-behavioral therapy versus education and desipramine versus placebo for moderate to severe functional bowel disorders. Gastroenterology 2003;125:19-31.

29. Creed F, Fernandes L, Guthrie E, Palmer S, Ratcliffe J, Read N, et al. The cost-effectiveness of psychotherapy and paroxetine for severe irritable bowel syndrome. Gastroenterology 2003;124:303-17.

30. Whorwell PJ. Review article: The history of hypnotherapy and its role in the irritable bowel syndrome. Aliment Pharmacol Ther 2005;22:1061-7.

31. Gonsalkorale WM, Whorwell PJ. Hypnotherapy in the treatment of irritable bowel syndrome. Eur $J$ Gastroenterol Hepatol 2005; 17:15-20.

32. McNeal S, Frederick C. Inner strength and other techniques for ego strengthening. American Journal of Clinical Hypnotherapy 1993:35:170-8.

33. Whitehead WE. Hypnosis for irritable bowel syndrome: the empirical evidence of therapeutic effects. Int $J$ Clin Exp Hypn 2006;54:7-20.

34. Lackner JM, Mesmer C, Morley S, Dowzer C, Hamilton S. Psychological treatments for irritable bowel syndrome: a systematic review and meta-analysis. J Consult Clin Psychol 2004;72:1100-13.

35. American College of Gastroenterology Functional Gastrointestinal Disorders Task Force. Evidence-based position statement on the management of irritable bowel syndrome in North America. Am J Gastroenterol 2002;97 [Suppl]:S1-S5.

36. Lea R, Whorwell PJ. Psychological influences on the irritable bowel syndrome. Minerva Med 2004;95:443-50.

37. Palsson OS, Drossman DA. Psychiatric and psychological dysfunction in irritable bowel syndrome and the role of psychological treatments. Gastroenterol Clin North Am 2005;34:281-303.

38. Creed F, Guthrie E, Ratcliffe J, Fernandes L, Rigby C, Tomenson B, et al. Does psychological treatment help only those patients with severe irritable bowel syndrome who also have a concurrent psychiatric disorder? Aust $N$ Z J Psychiatry 2005:39:807-15.

39. Lackner JM, Lou Coad M, Mertz HR, Wack DS, Katz LA, Krasner SS, et al. Cognitive therapy for irritable bowel syndrome is associated with reduced limbic activity, GI symptoms and anxiety. Behav Res Ther 2006;44:621-38.

40. Reibel DK, Creeson JM, Brainard GC, Rosenzweig S. Mindfulness based stress reduction and health-related quality of life in a heterogeneous patient population. Gen Hosp Psychiatry 2001;23:183-92.

41. Speca M, Carlson L, Goodey E, Angen M. A randomized wait-list controlled trail: the effects of a mindfulness meditation based stress reduction program on mood and symptoms of stress in cancer outpatients. Psychosom Med 2000;62:613-22.

42. Carlson LE, Ursuliak Z, Goodey E, Angen M, Speca M. The effects of a mindfulness meditation based stress reduction program on mood and symptoms of stress in cancer outpatients: six month follow-up. Supportive Care in Cancer 2001;9:112-23.

43. Jacobs DL, Quigley EM, Rikkers LF. Gastric compliance and motility in the portal hypertensive rat. J Investe Sur 1992;5:109-14.

44. Baer RA. Mindfulness training as a clinical intervention: a conceptual and empirical review. Clinical Psychology: Science and Practice 2003;10:125-43.

45. Teasdale JD, Segal AV, Williams JMG. Mindfulness training and problem formulation. Clinical Psychology: Science and Practice 2003;10:157-60.

46. Roemer L, Orsillo SM. Expanding Our Conceptualization of and Treatment for Generalized Anxiety Disorder: Integrating Mindfulness/Acceptance-Based Approaches with Existing Cognitive-Behavioral Models. Clinical Psychology: Science and Practice 2002;9:54-68.

47. Wells A. GAD. Metacognition and mindfulness: an information processing analysis. Clinical Psychology: Science and Practice 2002:9:95-100.

48. Kabat-Zinn J. An outpatient program in behavioral medicine for chronic pain patients based on the practice of mindfulness meditation: theoretical considerations and preliminary results. Gen Hosp Psychiatry 1982;4:33-47.

49. Keefer L, Blanchard EB. The effects of relaxation response meditation on the symptoms of irritable bowel syndrome: results 
of a controlled treatment study. Behavior Research and Therapy 2001;39:801-11.

50. Teasdale JD, Segal ZV, Williams JG, Mark G. How does cognitive therapy prevent depressive relapse and why should attentional control (mindfulness) training help? Behav Res Ther 1995;33:25-39.

51. Hayes SC, Luoma JB, Bond FW, Masuda A, Lillis J. Acceptance and commitment therapy: Model, processes and outcomes. Behav Res Ther 2006;44:1-25.

52. Fletcher L, Hayes SC. Relational frame theory, acceptance and commitment therapy and a functional analytic definition of mindfulness. $J$ Rational-Emotive \& Cognitive-Behavior Therapy 2005;23:315-36.

53. Hayes SC, Barnes-Holmes D. Relational operants: processes and implications: a response to Palmer's review of Relational Frame Theory. J ExperAnal Behav 2004;82:213-24.

54. McCracken LM. Learning to live with the pain: acceptance of pain predicts adjustment in persons with chronic pain. Pain 1998;74:21-7.
55. McCracken LM, Spertus IL, Janeck AS, Sinclair D, Wetzel FT. Behavioral dimensions of adjustment in persons with chronic pain: pain-related anxiety and acceptance. Pain 1999;80:283-9.

56. McCracken LM, Vowles KE, Eccleston C. Acceptance of chronic pain: component analysis and a revised assessment method. Pain 2004;107:159-66.

57. Dahl J, Wilson KG, Nilsson A. Acceptance commitment therapy for long term disability resulting from stress and pain symptoms: a preliminary randomized trial. Behav Ther 2004;35:785-801.

58. Bond F, Brunc D. Mediators of change in problem-focused and emotion-focused worksite stress management interventions. J Occup Heal Psyy 2000;5:156-63.

59. Salmon PG, Santorello SF, Kabat-Zinn J. Intervention elements in promoting adherence to mindfulness based stress reduction in the clinical behavioral medicine setting. In: Schumake SA, Schron EB, Ockene JK, (eds). The handbook of health behavior change. New York, USA: Springer, 1998, 239-66.

Received March 7, 2006; accepted April 4, 2007 


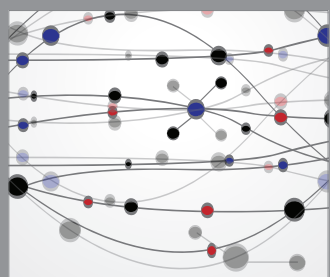

The Scientific World Journal
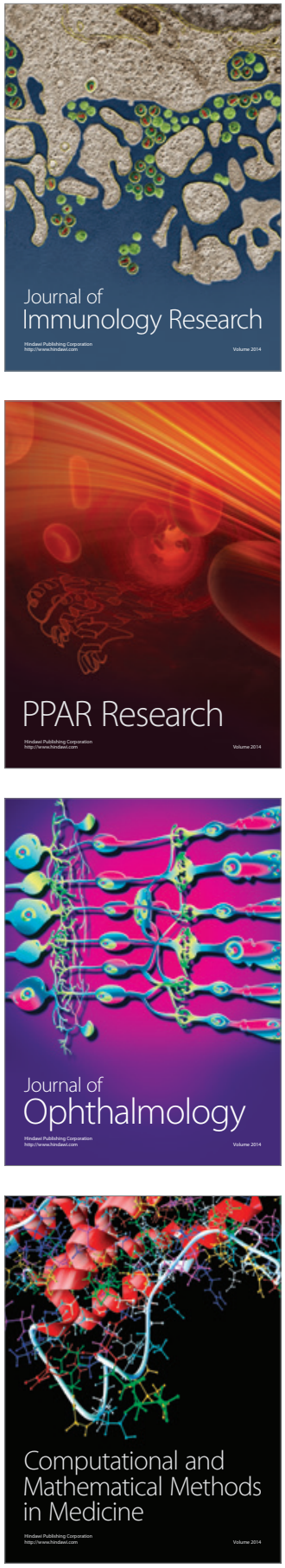

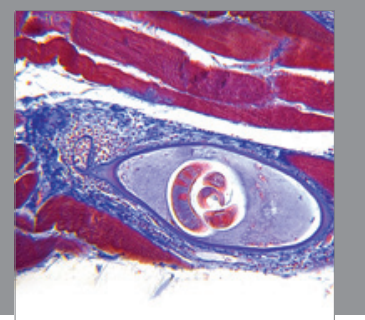

Gastroenterology

Research and Practice
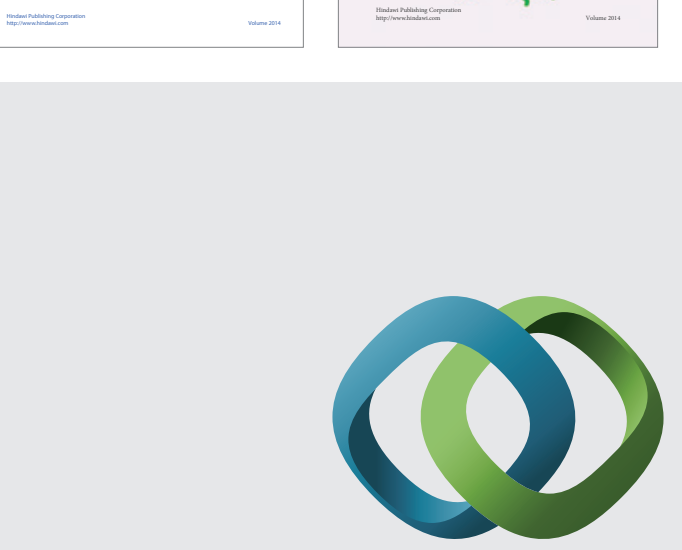

\section{Hindawi}

Submit your manuscripts at

http://www.hindawi.com
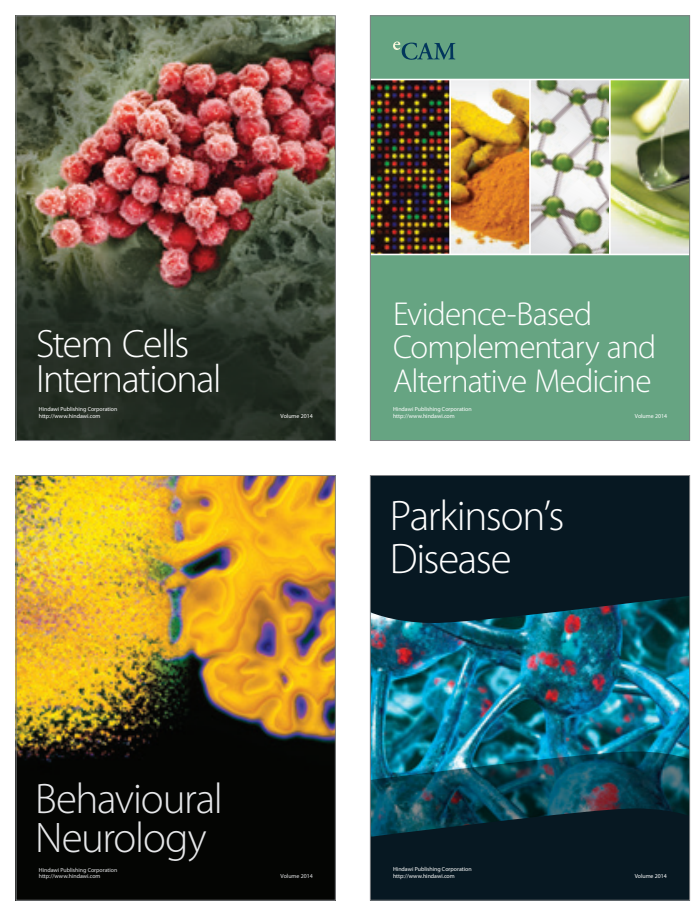

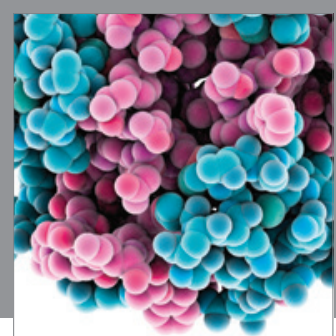

Journal of
Diabetes Research

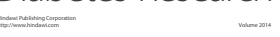

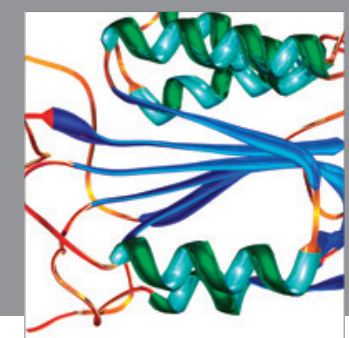

Disease Markers
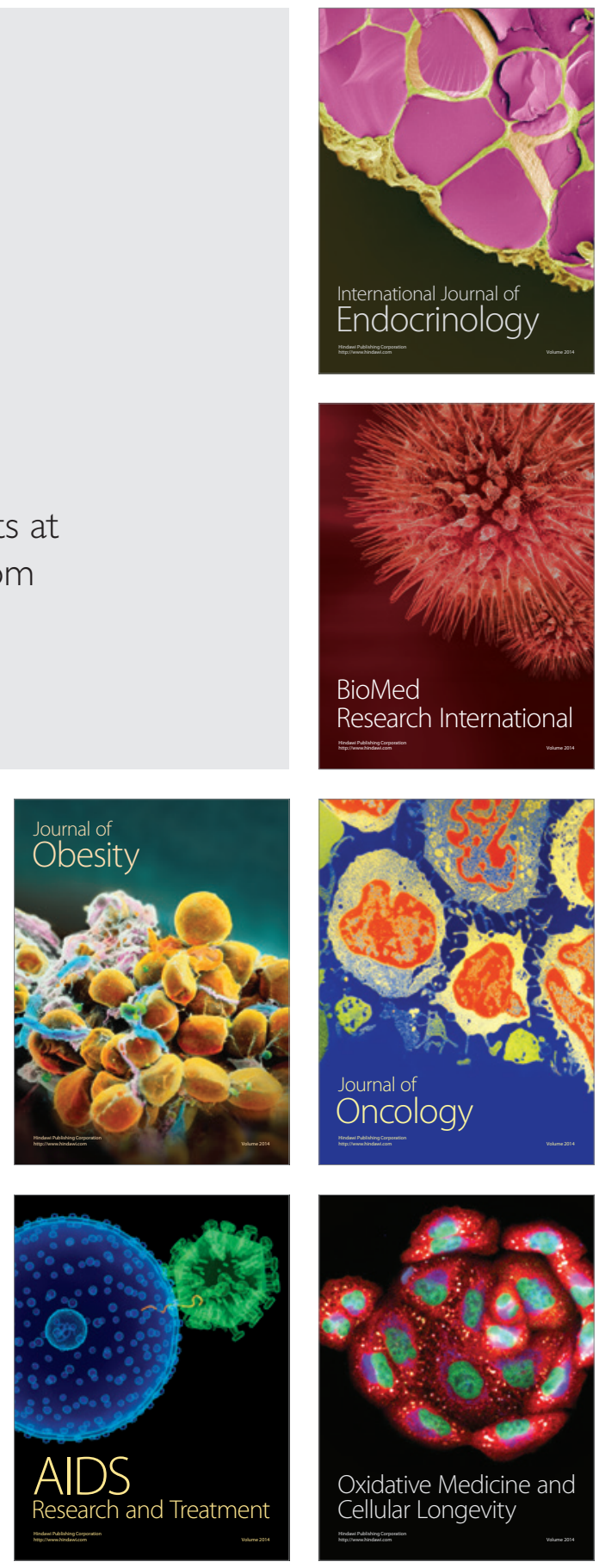